\title{
miR-122 promotes hepatic lipogenesis via inhibiting the LKB1/AMPK pathway by targeting Sirt1 in non-alcoholic fatty liver disease
}

\author{
Jun-Ke Long ${ }^{1}$, Wen Dai ${ }^{1}$, Ya-Wen Zheng ${ }^{2}$ and Shui-Ping Zhao ${ }^{1 *}$ (D)
}

\begin{abstract}
Background: Non-alcoholic fatty liver disease (NAFLD) is a common hepatic disease with an increasing prevalence but an unclear aetiology. This study aimed to investigate the functional implications of microRNA-122 (miR-122) in the pathogenesis of NAFLD and the possible molecular mechanisms.

Methods: Both in vitro and in vivo models of NAFLD were generated by treating HepG2 and Huh-7 cells with free fatty acids (FFA) and by feeding mice a high-fat diet (HFD), respectively. HE and Oil Red O staining were used to examine liver tissue morphology and lipid deposition, respectively. Immunohistochemical (IHC) staining was used to examine Sirt1 expression in liver tissues. qRT-PCR and Western blotting were employed to measure the expression of miR-122, Sirt1, and proteins involved in lipogenesis and the AMPK pathway. Enzyme-linked immunosorbent assay (ELISA) was used to quantify triglyceride (TG) levels in HepG2 and Huh-7 cells and in liver tissues. The interaction between miR-122 and the Sirt1 gene was further examined by a dual luciferase reporter assay and RNAimmunoprecipitation (RIP).

Results: NAFLD hepatic tissues and FFA-treated HepG2 and Huh-7 cells presented excess lipid production and TG secretion, accompanied by miR-122 upregulation, Sirt1 downregulation, and potentiated lipogenesis-related genes. miR-122 suppressed Sirt1 expression via binding to its 3'-untranslated region (UTR). Knockdown of miR122 effectively mitigated excessive lipid production and suppressed the expression of lipogenic genes in FFAtreated HepG2 and Huh-7 cells via upregulating Sirt1. Furthermore, miR-122 knockdown activated the LKB1/ AMPK signalling pathway.

Conclusion: The inhibition of miR-122 protects hepatocytes from lipid metabolic disorders such as NAFLD and suppresses lipogenesis via elevating Sirt1 and activating the AMPK pathway. These data support miR-122 as a promising biomarker and drug target for NAFLD.
\end{abstract}

Keywords: miR-122, Non-alcoholic fatty liver disease, Lipogenesis, Sirt1, AMPK pathway

\footnotetext{
* Correspondence: zhaoshuiping03@163.com

1Department of Cardiovascular Medicine, The Second Xiangya Hospital of

Central South University, No.139, Middle Renmin Road, Changsha 410011,

Hunan Province, People's Republic of China

Full list of author information is available at the end of the article
}

(c) The Author(s). 2019 Open Access This article is distributed under the terms of the Creative Commons Attribution 4.0 International License (http://creativecommons.org/licenses/by/4.0/), which permits unrestricted use, distribution, and reproduction in any medium, provided you give appropriate credit to the original author(s) and the source, provide a link to the Creative Commons license, and indicate if changes were made. The Creative Commons Public Domain Dedication waiver (http://creativecommons.org/publicdomain/zero/1.0/) applies to the data made available in this article, unless otherwise stated. 


\section{Background}

Nonalcoholic fatty liver disease (NAFLD) is a prevalent chronic disease worldwide and has become an emerging health threat in recent years (Spengler \& Loomba, 2015). Epidemiological surveys have shown that the incidence of NAFLD is sharply increasing, mainly due to high-fructose diet ingestion and higher rates of obesity and diabetes (Dai et al., 2017a). The timely intervention of NAFLD is thus of critical importance for reducing public health burdens. Currently, major therapeutic strategies for NAFLD consist of lifestyle adjustment and medication that improve lipid homeostasis. However, the associated side effects of existing drugs, such as weight gain or higher long-term mortality, still restrain the medical treatment of NAFLD (Takahashi et al., 2015). Therefore, the identification of novel biomarkers is critical for both early diagnosis and the precise intervention of NAFLD.

microRNAs (miRNAs), which are small non-coding RNA molecules, can mediate metabolic homeostasis via unique post-transcriptional regulatory mechanisms (Rottiers \& Naar, 2012). A role of miRNA in NAFLD has been suggested recently. Accumulating evidence has revealed the unique miRNA expression profiles that are tightly correlated with NAFLD conditions (Panera et al., 2014). For example, a recent study involving 47 NAFLD patients found that circulating levels of miR-122, miR-192 and miR-375 were upregulated and were positively correlated with disease severity (Pirola et al., 2015). In a rat NAFLD model, miR-122 has also been shown to be affected (Yamada et al., 2015). As one of the most abundant miRNAs in the liver,miR-122 was significantly upregulated in serum and was suggested as a potential biomarker for NAFLD in patients (Jampoka et al., 2018; Ye et al., 2018; Akuta et al., 2016). In particular, miR-122 upregulation was found to be directly associated with the severity of liver fibrosis in NAFLD patients (Miyaaki et al., 2014). It was thus speculated that miR-122 might function as a predictive factor for disease progression for NAFLD (Ceccarelli et al., 2013). However, the detailed molecular mechanism of miR-122 in NAFLD is still unclear.

Sirtuin 1 (Sirt1) is a nicotinamide adenine dinucleotide (NAD)-dependent deacetylase that has been implicated in NAFLD progression (Nassir \& Ibdah, 2016). A previous study reported that Sirt1 expression was downregulated in liver tissues of a rat NAFLD model (Deng et al., 2007). Moreover, the knockout of the Sirt1 gene in hepatocytes suppressed fatty acid oxidation (Purushotham et al., 2009). D. Herranz et al. revealed that Sirt1 could protect liver tissues from ageing-related metabolic stress (Herranz et al., 2010). Mechanistically, Sirt1 regulates cellular metabolism via NAD-dependent histone deacetylation to affect transcriptional networks (Imai et al.,
2000). Sirt1 also modulates the expression of various genes involved in glucose and lipid metabolism ( $\mathrm{Li}$, 2013). Upstream of Sirt1, its expression is regulated by various non-coding RNAs, such as miR-34a (Li et al., 2015). However, the relationship between Sirt1 and miR-122 in NAFLD remains elusive.

In this study, we performed both in vitro and in vivo experiments to examine the function of miR-122 on Sirt1-mediated lipid metabolism in NAFLD pathogenesis. We found that miR-122 was upregulated both in cell lines and in an animal model of NAFLD; by contrast, Sirt1 expression was downregulated. A mechanistic study revealed that miR-122 directly bound to the 3'-UTR of Sirt1 to suppress its expression. Knockdown of miR-122 decreased the expression of lipogenic genes but activated the AMPK signalling pathway, which further suppressed lipid production and triglyceride (TG) secretion in hepatocytes. Our results thus provide the novel insight into NAFLD pathogenesis and highlight the potency of miR-122 as both a biomarker and therapeutic target for NAFLD in the future.

\section{Methods}

\section{Cell culture and treatment}

The human hepatocyte cell lines HepG2 and Huh-7 were obtained from the American Type Culture Collection (ATCC, US) and cultured in a humidified incubator at $37^{\circ} \mathrm{C}$ with $5 \% \mathrm{CO}_{2}$ using Dulbecco's Modified Eagle Medium (DMEM) (Gibco, US), which was supplemented with $1 \%$ penicillin and streptomycin (Invitrogen, US) and $10 \%$ foetal bovine serum (FBS) (Gibco, US). To establish the in vitro NAFLD cell model, HepG2 and Huh-7cells were cultured in the presence or absence of $1 \mathrm{mM}$ free fatty acids (FFA, containing oleic acid and palmitic acid at a 2:1 volume ratio) for $24 \mathrm{~h}$ and then used for the indicated assays.

\section{NAFLD mouse model}

The NAFLD mouse model was developed according to previously reported protocols (Soares e Silva et al., 2015). In brief, a total of 16 C57BL/6 mice (males, 8 weeks old) were purchased from SJA Laboratory Animal Corp (Changsha, China) and were randomly assigned to the high-fat diet (HFD) or the standard chow diet (SCD) group ( $n=8$ in each group). Mice were then fed the indicated food chow for 8 weeks to establish the NAFLD model. All animal experimental procedures followed the guidelines of the Ethical Committee of the Second Xiangya Hospital, Central South University.

\section{ELISA assay}

The TG contents in HepG2 and Huh-7 cells or liver tissues isolated from the NAFLD mouse model were measured with an ELISA assay. Briefly, HepG2 and Huh-7 
cells were pre-treated with FFA, and then the cell lysates and the supernatant were collected for TG quantification. For NAFLD mice, liver tissues were collected after sacrifice and then homogenized and lysed in $5 \% \mathrm{NP}-40 / \mathrm{dd}_{2} \mathrm{O}$ solution. After centrifugation, the supernatant was collected for subsequent TG quantification. The concentration of TG was measured using ELISA kits (Abcam, UK) according to the manufacturer's instructions.

\section{Cell transfection}

The sequences of the shRNA targeting Sirt1 (shSirt1) and the negative control shRNA (shNC) were 5' - CCGGGATGATCAAGAGGCAATTAATCTCGAGATTAATT GCCTCTTGATCATCTTTTTG-3' and 5' ${ }^{\prime}$-CCGG CCTAAGGTTAAGTCGCCCTCGCTCGAGCGAGGGC GACTTAACCTTAGGTTTTTG-3', respectively. shSirt1 and shNC were subcloned into the pSicoR plasmid vector, and sequence-verified plasmids were then used for subsequent transfection. miR-122 mimics, inhibitors and negative control miRNAs were purchased from GenePharma (Shanghai, China). Cell transfection and cotransfection were conducted with Lipofectamine 2000 (Invitrogen, USA) according to the manufacturer's instruction. In brief, $5 \mu \mathrm{L}$ of Lipofectamine-2000 reagent was diluted into 0.25 $\mathrm{mL}$ of OPTI-MEM medium, while $5 \mu \mathrm{L}$ of miR-122 mimics/inhibitor/negative control miRNA (100 pmol) or $4.0 \mu \mathrm{g}$ of $\mathrm{pSicoR}-$ Sirt $1 / \mathrm{pSicoR}$ plasmid was added to another $0.25 \mathrm{~mL}$ aliquot of OPTI-MEM medium. Then, they were incubated separately for $5 \mathrm{~min}$ at room temperature. After that, they were combined in one tube, gently inverted and incubated for $20 \mathrm{~min}$. Subsequently, $0.5 \mathrm{~mL}$ of the mixture was added to the HepG2 and Huh-7 cells drop wise. The plate was then swirled several times to ensure that the mixture was distributed evenly. After culturing for $6 \mathrm{~h}$, the medium was replaced with fresh DMEM medium containing 10\% FBS. The cells were then cultured for another $48 \mathrm{~h}$ before the subsequent experiments.

\section{Dual luciferase reporter assay}

A dual luciferase reporter assay was employed to examine the interaction between Sirt1 and miR-122 in HepG2 and Huh-7 cells according to a previously reported method (Hu et al., 2014). Specifically, wild-type and mutant (without miR-122 binding sites) three prime untranslated regions (3'-UTRs) from Sirt1 mRNAs were cloned and inserted downstream of the luc2 firefly luciferase in the pmiRGLO vector (Promega, US), which was then co-transfected with miR-122 mimics into HepG2 and Huh-7 cells. The constitutively expressed Renilla luciferase in the pmiRGLO vector provided a normalization reference. After $48 \mathrm{~h}$ of transfection, the HepG2 and Huh-7 cells were lysed and subjected to luciferase activity measurement with the Dual Luciferase Assay Kit (Promega, US) according to the manufacturer's instructions. The firefly and Renilla luciferase activities were determined by a plate reader (NEO, Bio-Tek, USA) and normalized to the Renilla luciferase data.

\section{RNA immunoprecipitation (RIP)}

We used an RIP assay to directly confirm the interaction between miR-122 and Sirt1 according to a method in a previous report (Chen et al., 2018). Briefly, HepG2 and Huh-7 cells were lysed in $300 \mu \mathrm{L}$ of lysis buffer supplemented with RNase inhibitor and complete protease inhibitor for $20 \mathrm{~min}$ on ice, and then the cell lysate was centrifuged for $10 \mathrm{~min}$ at $4{ }^{\circ} \mathrm{C}$. The supernatant was collected and pre-cleared with protein A-Sepharose beads. Next, the supernatant was incubated with mouse anti-human Ago2 antibody (Cell Signaling Technology, USA) or negative control antibody (normal mouse IgG, Cell Signaling Technology, USA) for $4 \mathrm{~h}$, followed by the addition of protein $\mathrm{A} / \mathrm{G}$ sepharose beads and incubation for $2 \mathrm{~h}$. The beads were then rinsed with NT2 buffer supplemented with RNase inhibitor and complete protease inhibitors, and bead-captured protein-RNA complexes were digested using DNase I and proteinase $\mathrm{K}$ and were then eluted by NT2 buffer. RNA was finally extracted by the phenol-chloroform method and quantified by qRT-PCR. The primers used for amplifying the Sirt1 were as follows: forward: 5 '-TTTGTCAGAGTTGCCACCCA-3'; reverse: 5' -GCCGCCTACTAATCTGCTCC-3'.

\section{RNA extraction and quantitative real-time PCR (qRT-PCR)}

qRT-PCR was used to quantify the relative expression levels of the miR-122, Sirt1, sterol regulatory element-binding protein 1(SREBP1), stearoyl-CoA desaturase 1(SCD1), acetyl-coA carboxylase (ACC1), fatty acid synthase (FASN) and apolipoprotein A5 (ApoA5) genes in both mouse liver tissues and cultured hepatocytes. In brief, total RNA including small RNA was extracted from liver tissues, HepG2 and Huh-7 cells using the miRNeasy Mini Kit (Qiagen, Germany) according to the manufacturer's instructions. The first-strand cDNA was synthesized via in vitro reverse transcription (RT) from total RNA by using the QuantiTect Reverse Transcription Kit (Qiagen, Germany) or from small RNA by using the miScript II RT Kit (Qiagen, Germany). qRT-PCR was then performed on an Applied Biosystems $^{\mathrm{Tx}}$ QuantStudio $^{\mathrm{Tm}} 6$ Flex Real-Time PCR System (Thermo Fisher Scientific, Carlsbad, CA, USA) with the SYBR Green method. PCR was performed under the following conditions: $95^{\circ} \mathrm{C}$ for $10 \mathrm{~min}$, followed by $40 \mathrm{cy}$ cles each of $95^{\circ} \mathrm{C}$ for $15 \mathrm{~s}, 60^{\circ} \mathrm{C}$ for $20 \mathrm{~s}$ and $72^{\circ} \mathrm{C}$ for $40 \mathrm{~s}$. The $\mathrm{Ct}$ values were calculated, and the relative expression level was quantified by the $2^{-\Delta \Delta \mathrm{Ct}}$ approach using $\beta$-actin and U6 as the normalization references for mRNA and miRNA, respectively. Each sample was 
tested in triplicate for statistical analysis. The primers used in qRT-PCR are listed in Table 1.

\section{Western blotting}

Western blotting was used to measure the expression levels of Sirt1, liver kinase B1 (LKB1), AMP-activated protein kinase (AMPK) and p-AMPK proteins both in cell lines and in mouse liver tissues. In brief, cells or tissues were lysed and homogenized in RIPA buffer (50 $\mathrm{mM}$ Tris- $\mathrm{HCl}, 150 \mathrm{mM} \mathrm{NaCl}, 1 \% \mathrm{NP}-40,0.5 \%$ sodium deoxycholate, and $0.1 \%$ SDS, pH 7.4) supplemented with

Table 1 Primers used for qRT-PCR analysis

\begin{tabular}{|c|c|}
\hline Genes & Primer sequences $\left(5^{\prime}-3^{\prime}\right)$ \\
\hline \multirow[t]{2}{*}{ hsa(mmu)-miR-122 } & F: 5'-AGCGTGGAGTGTGACAATGG-3' \\
\hline & $\begin{array}{l}\text { R: 5'-GTCGTATCCAGTGCAGGGTCCGAG } \\
\text { GTATTCGCACTGGATACGACCAAACA-3' }\end{array}$ \\
\hline \multirow[t]{2}{*}{ hsa(mmu)-U6 } & F: 5'-CTCGCTTCGGCAGCACA-3' \\
\hline & R: 5'-AACGCTTCACGAATTTGCGT-3' \\
\hline \multirow[t]{2}{*}{ hSirt1 } & F: 5'-TGCCGGAAACAATACCTCCA-3' \\
\hline & R: 5'-AGACACCCCAGCTCCAGTTA-3' \\
\hline \multirow[t]{2}{*}{ hSREBP1 } & F: 5'-GGAGCCATGGATTGCACTTTCG-3' \\
\hline & R: 5'-GCTCAGGAAGGCTTCAAGAGAG-3' \\
\hline \multirow[t]{2}{*}{ hFASN } & F: 5'-TATGAAGCCATCGTGGACGG-3' \\
\hline & R: 5'-GAAGAAGGAGAGCCGGTTGG-3' \\
\hline \multirow[t]{2}{*}{ hSCD1 } & F: 5'-CTTGCGATATGCTGTGGTGC-3' \\
\hline & R: 5'-AAGTTGATGTGCCAGCGGTA-3' \\
\hline \multirow[t]{2}{*}{ hACC1 } & F: 5'-CAAGGTCAGCTGGTCCACATG-3' \\
\hline & R: 5'-GTGGAATACCTTCTGCCCTAGC-3' \\
\hline \multirow[t]{2}{*}{ hApoA5 } & F: 5'- GACCAGGAGACTGAGGAGGT-3' \\
\hline & R: 5'-TTGCTCAGAACCTTGCCACT-3' \\
\hline \multirow[t]{2}{*}{$h \beta$-actin } & F: 5'- CCTCGCCTTTGCCGATCC-3' \\
\hline & R: 5'- GGATCTTCATGAGGTAGTCAGTC - 3' \\
\hline \multirow[t]{2}{*}{ mSirt1 } & F: 5'-GACGCTGTGGCAGATTGTTA-3' \\
\hline & R: 5'-GGAATCCCACAGGAGACAGA-3' \\
\hline \multirow[t]{2}{*}{ mSREBP1 } & F: 5'-CCACAATGCCATTGAGAAGCG-3' \\
\hline & R: 5'-CTGACACCAGGTCCTTCAGTG-3' \\
\hline \multirow[t]{2}{*}{ mFASN } & F: 5'-TTGCTGGCACTACAGAATGC-3' \\
\hline & R: 5'-AACAGCCTCAGAGCGACAAT-3' \\
\hline \multirow[t]{2}{*}{ mSCD1 } & F: 5'-CACACCTTCCCCTTCGACTA-3' \\
\hline & R: 5'-TGACTCCCGTCTCCAGTTCT-3' \\
\hline \multirow[t]{2}{*}{ mACC1 } & F: 5'-CTTGGAGCAGAGAACCTTCG-3' \\
\hline & R: 5'-ACTTCCCGACCAAGGACTTT-3' \\
\hline \multirow[t]{2}{*}{ mApoA5 } & F: 5'-AGAAGCTGGCACAGGAGAAC-3' \\
\hline & R: 5'-AGCTGAGCCTTGGTGTCTTC-3' \\
\hline \multirow[t]{2}{*}{$m \beta$-actin } & F: 5'-GTCGTACCACAGGCATTGTGATGG-3' \\
\hline & R: 5'-GCAATGCCTGGGTACATGGTGG-3' \\
\hline
\end{tabular}

Note: The primers were manufactured by Nanjing Genescript Co. Ltd. (Nanjing, P.R China)
$1 \times$ protease inhibitor cocktail, and the protein contents in the cell lysates were quantified by a BCA kit (Beyotime, China). Approximately $30 \mu \mathrm{g}$ of protein sample was subjected to SDS-PAGE separation and was then transferred to a PVDF membrane (Millipore Corp, Bedford, MA). After blocking with 5\% BSA in Tris-buffered saline with $0.1 \%$ Tween 20 (TBST), the PVDF membrane was rinsed and incubated with primary antibody (rabbit anti-Sirt1 antibody at 1:1000, rabbit anti-LKB1 antibody at 1:2000, rabbit anti-AMPK antibody at 1:1500, and rabbit anti-p-AMPK antibody and rabbit anti-GAPDH antibody at 1:1000, Cell Signaling Technology, USA) at $4{ }^{\circ} \mathrm{C}$ overnight. After vigorous washing with TBST 3 times, the PVDF membrane was then incubated with goat anti-rabbit IgG-HRP antibody (1:5000, Cell Signaling Technology, USA) for $1 \mathrm{~h}$ at room temperature. After washing with TBST, the membrane was then incubated with ECL substrate, and images were captured by a ChemiDoc MP imaging system (Bio-Rad, USA).

\section{Oil red $O$ staining}

Oil Red $\mathrm{O}$ staining was applied to assess lipid droplet formation in HepG2 and Huh-7 cells and liver tissues according to a previously described method (Chu et al., 2014). First, HepG2 and Huh-7 cells were pre-cultured on coverslips and were then washed with PBS and fixed in $10 \%$ formalin for $5 \mathrm{~min}$. After fixation, the cells were briefly rinsed in isopropanol and incubated with Oil Red $O$ reagent for $30 \mathrm{~min}$. The stained cells were then washed in distilled water and counter-stained with haematoxylin for $1 \mathrm{~min}$. The mouse liver tissues were first fixed in $4 \%$ paraformaldehyde and then embedded in paraffin and sectioned into slices with $6-\mu \mathrm{m}$ thickness. These sections were then de-waxed in xylene and rehydrated. After rinsing with PBS, the tissue sections were incubated in Oil Red $\mathrm{O}$ reagent for $30 \mathrm{~min}$, followed by haematoxylin counter-staining for $1 \mathrm{~min}$. After washing and dehydration, the Oil Red $\mathrm{O}$ and haematoxylin-stained sections were mounted for fluorescence microscope (IX-51, Olympus) for imaging. Five randomly selected images were taken for each sample. Data were collected from at least three independent experiments.

\section{HE staining of liver tissues}

Haematoxylin-eosin (HE) staining was performed to examine liver tissue morphology according to standard protocols. In brief, liver tissues were fixed with $4 \%$ paraformaldehyde (PFA) overnight. The fixed tissue samples were then embedded in paraffin and sectioned into slices with $6 \mu \mathrm{m}$ thickness, which were then dehydrated with different concentrations of ethanol and xylol followed by brief washing and staining of cell nuclei with 5\% 
haematoxylin solution for $10 \mathrm{~min}$. After rinsing in distilled water for $5 \mathrm{~min}$, the stained samples were incubated in $0.1 \% \mathrm{HCl}$-ethanol for $30 \mathrm{~s}$. The samples were then counterstained with eosin solution for $2 \mathrm{~min}$. After washing and dehydration, the HE-stained sections were mounted for fluorescence microscope (IX-51, Olympus) for imaging.

\section{Immunohistochemistry (IHC)}

IHC was used to measure the expression of Sirt1 protein in mouse liver tissues. In brief, liver tissues were fixed with 4\% PFA and embedded in paraffin. The samples were then sectioned into slices with a thickness of $6 \mu \mathrm{m}$, which were then attached to slides, washed and incubated with $3 \% \mathrm{H}_{2} \mathrm{O}_{2}$ in methanol for 10 min to block endogenous peroxidase activity. Afterwards, these sections were blocked and then incubated with a primary antibody against Sirt1 (1:500, Abcam) at $4{ }^{\circ} \mathrm{C}$ overnight. After washing with $\mathrm{PBS}$, the sections were incubated with secondary antibody for $1 \mathrm{~h}$ at room temperature. Finally, the sections were washed and incubated with DAB substrate for visualization. After washing and dehydration, the sections were sealed with coverslips and subjected to fluorescence microscopy (IX-51, Olympus) for imaging.

\section{Statistical analysis}

All data are presented as the mean \pm standard deviation (SD). Student's $t$-test was used to compare differences between two groups, while one-way analysis of variance (ANOVA) was performed to compare differences among multiple groups, which was followed by Tukey's post hoc comparison test. Statistical significance was indicated when $p<0.05$. All statistical analyses were performed using SPSS18.0 software.

\section{Results}

\section{Elevated miR-122 expression and decreased Sirt1} expression in the NAFLD mouse model

To explore the relationship between Sirt1 and miR-122 in NAFLD, we first generated a mouse model of NAFLD using HFD feeding for 8 weeks. HE staining of liver tissues showed that there were increased hepatocyte volumes and more dispersed lipid vacuoles and compressed liver sinusoids in NAFLD mice compared to those in control mice (Fig. 1a). Consistently, Oil Red O staining results showed remarkable deposition of lipid droplets within the liver in NAFLD mice (Fig. 1a). Furthermore, an elevated TG secretion level was also identified in NAFLD mice (Fig. 1b). In addition, we further investigated the expression of miR-122 in mouse liver tissues. To our surprise, we found that it was upregulated in the liver of NAFLD mice (Fig. 1c). In addition, we examined multiple genes involved in hepatic lipogenesis, such as Sirt1, SREBP1, FASN, SCD1, ACC1 and ApoA5, by
qRT-PCR. The data showed that only Sirt1 expression was reduced, while the expression of the other genes was elevated in NAFLD mice (Fig. 1c). Moreover, both Western blotting (Fig. 1d and e) and IHC staining (Fig. If and g) confirmed the decreased Sirt1 protein expression in liver tissues of NAFLD mice. In summary, these experiments revealed that Sirt1 was downregulated, while miR-122 and other lipogenic genes were all upregulated in the livers of NAFLD mice.

\section{FFA induces miR-122 upregulation and lipogenesis in hepatocytes}

To examine the molecular pathway downstream of miR-122, we used two commonly used hepatocyte cell lines, HepG2 and Huh-7, for the in vitro cellular experiments. To mimic the NAFLD phenotype, HepG2 and Huh-7 cells were treated with FFA for $24 \mathrm{~h}$. As expected, Oil Red O staining showed that FFA treatment remarkably induced lipid deposition inside cells (Fig. 2a). Moreover, FFA challenge robustly elevated TG secretion from HepG2 and Huh-7 cells (Fig. 2b). Consistent with NAFLD mice, both cell lines displayed elevated miR-122 expression but decreased Sirt1 expression as evidenced by qRT-PCR (Fig. 2c and d). In addition, Western blotting results confirmed that the protein level of Sirt1 was attenuated in FFA-treated HepG2 and Huh-7 cells (Fig. 2e and f). Overall, these data indicated that miR-122 might be involved in regulating FFA-induced lipogenesis and was negatively correlated with Sirt1 expression.

\section{miR-122 suppressesSirt1 expression via binding to the 3'-UTR of Sirt1}

To further study the relationship between Sirt1 and miR-122, we next transfected HepG2 and Huh-7 cells with the miR-122 mimics, the miR-122 inhibitor or the negative control (NC). qRT-PCR results showed that the miR-122 mimics remarkably decreased Sirt1 expression; however, the miR-122 inhibitor potently suppressed miR-122 expression but elevated Sirt1 expression in both HepG2 and Huh-7 cells (Fig. 3a). These data indicated that the Sirt1 gene was possibly negatively regulated by miR-122 in hepatocytes. To further confirm the association of these factors, we performed a bioinformatic prediction and found putative binding sites of miR-122 in the $3^{\prime}$-UTR of Sirt1 (Fig. 3b). To verify this prediction, we then executed RIP assay, and the results strongly supported the direct interaction between miR-122 and Sirt1, which was suggested by the significant enrichment of Sirt1 mRNA when miR-122 was overexpressed in HepG2 and Huh-7 cells (Fig. 3c). Furthermore, we applied a dual luciferase assay to measure the binding between the miR-122 mimics or inhibitors and the 3'-UTR of Sirt1. Consistently, the results showed 

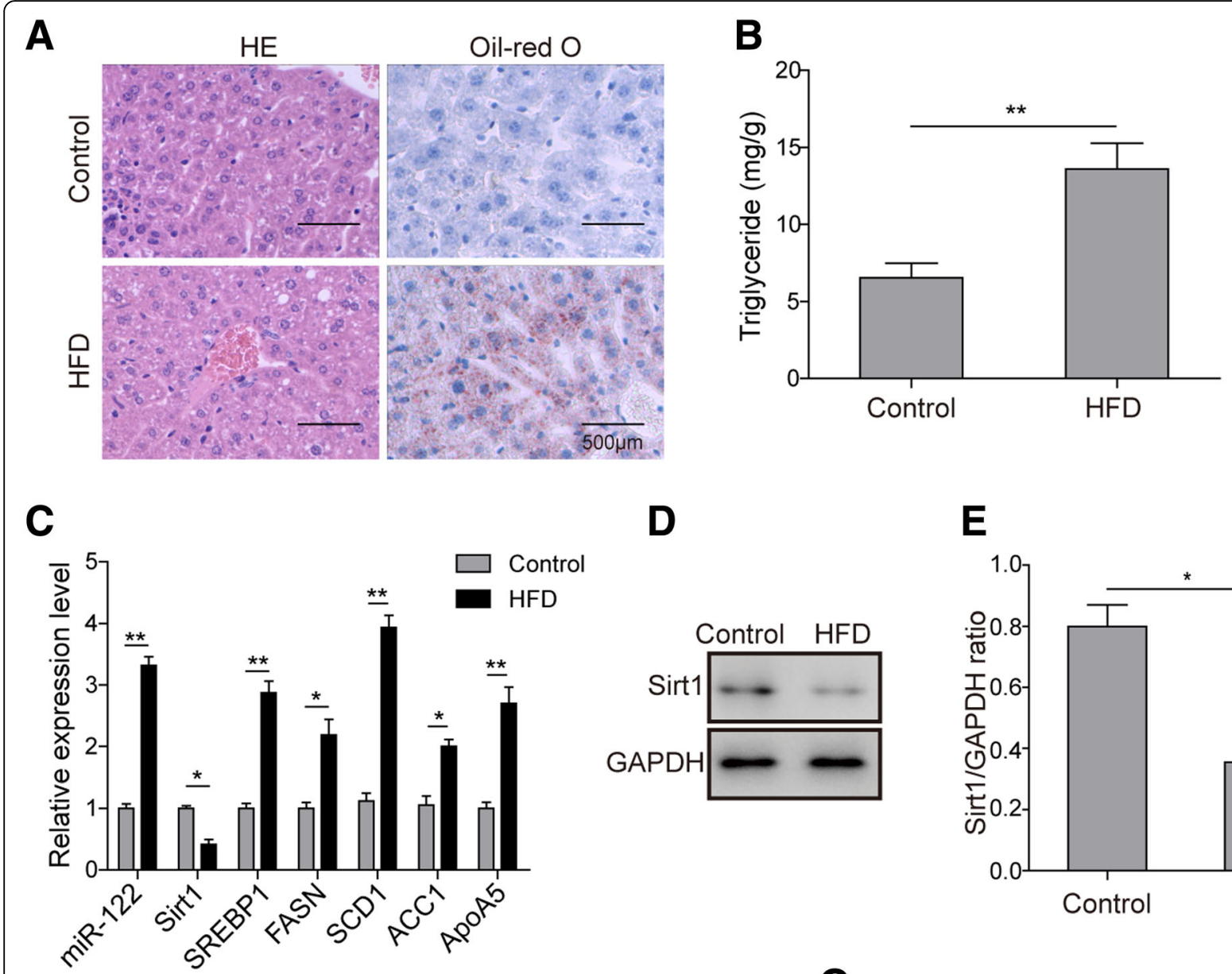

D

E
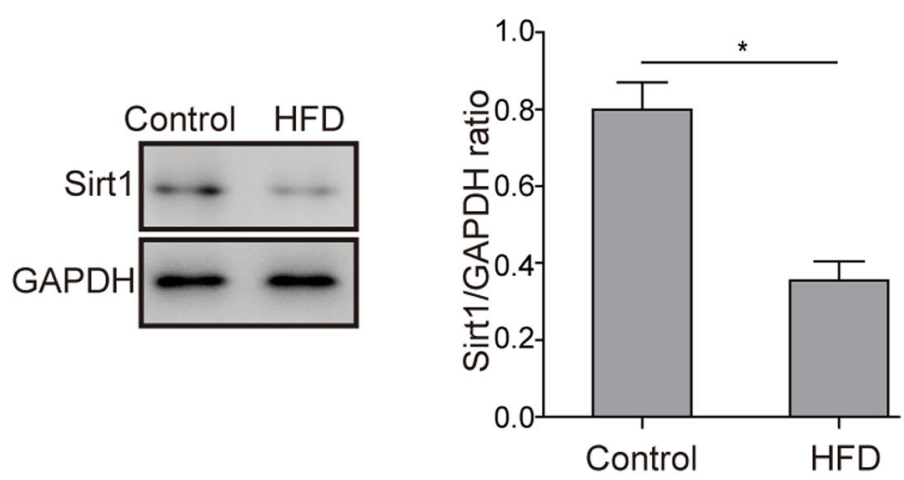

$\mathbf{F}$
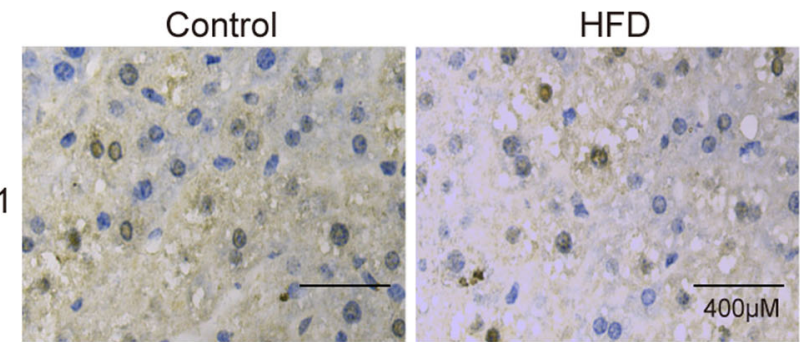

G

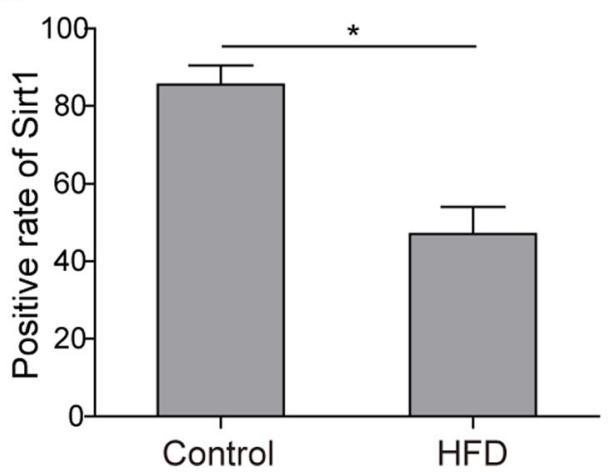

Fig. 1 Lipid pathology and altered gene expression in NAFLD mice. a HE staining (left) and Oil Red O staining (right) showing lipid accumulation in liver tissues. b Hepatic TG levels were assessed by ELISA. c The relative expression levels of miR-122, Sirt1 and lipogenesis-related genes in control diet- and high-fat diet (HFD)-fed mice were detected by qRT-PCR. $\mathbf{d}$ The expression level of Sirt1 was measured by Western blotting. $\mathbf{e}$ Quantification of the Western blotting results in $\mathbf{d} . \mathbf{f}, \mathbf{g}$ Representative images $(\mathbf{f})$ and thepositive rate $(\mathbf{g})$ of Sirt 1 expression in the liver tissues of NAFLD mice as revealed by $\mathrm{IHC}$ staining. All the results are shown as the mean $\pm \mathrm{SD}(n=3)$, which are representative of three independent experiments performed in triplicate. ${ }^{*} p<0.05$ and ${ }^{* *} p<0.01$

that the miR-122 mimics, but not the NC, clearly repressed luciferase activity, and this suppression was completely abolished when the binding sites in the 3 '-UTR of Sirt1 were mutated (Fig. 3d). Overall, these results indicated that miR-122 participated in the modulation of Sirt1 via binding to the 3'-UTR of Sirt1.
Knockdown of miR-122 alleviates lipid deposition by directly upregulating Sirt1 in hepatocytes

After revealing the association between miR-122 and Sirt1, we next investigated the influence of this interaction on lipid metabolic disorder in hepatocytes by knocking down miR-122 or Sirt1. First, we transfected 


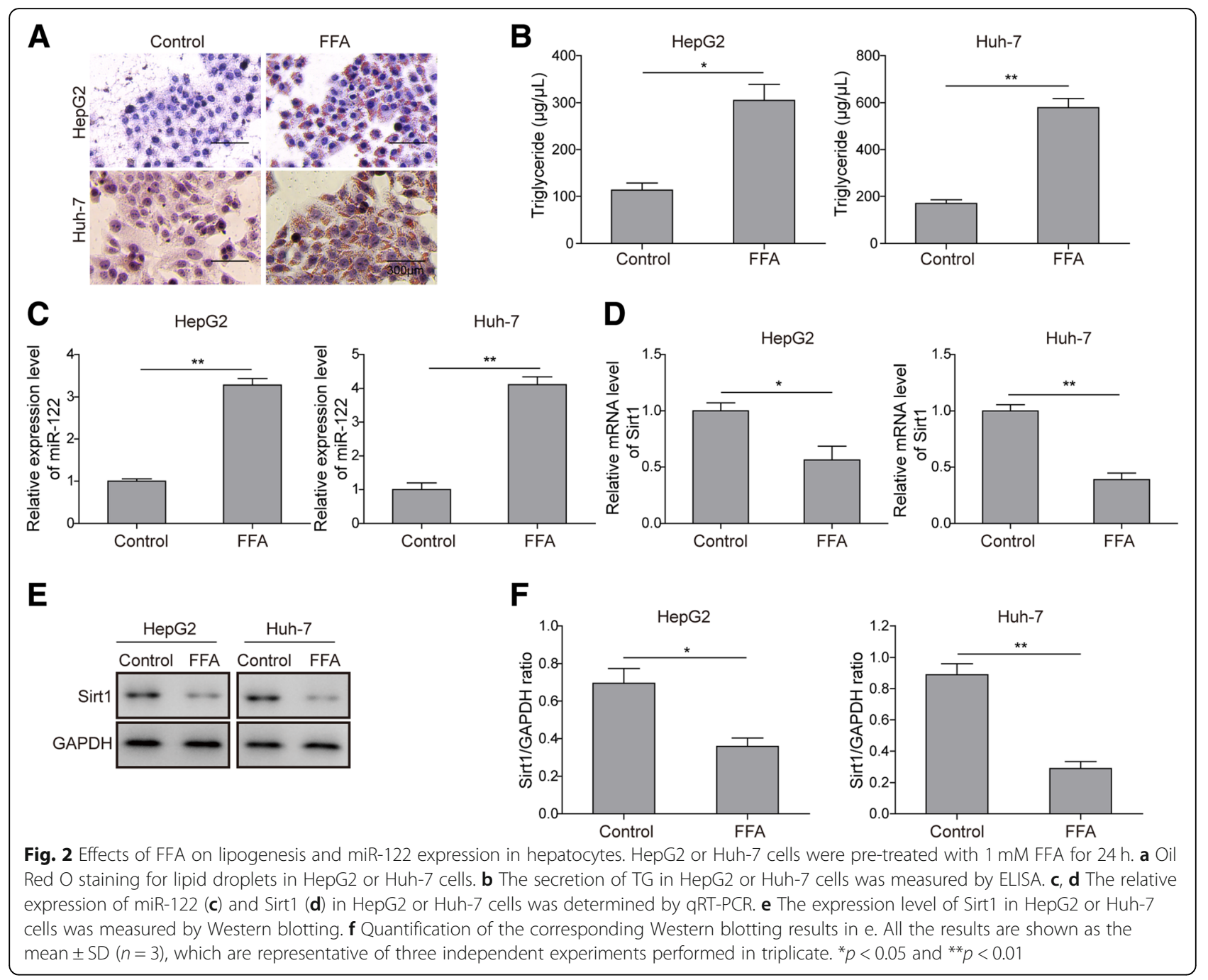

shSirt1 into HepG2 or Huh-7 cells, and subsequent qRT-PCR and Western blotting results showed that the expression level of Sirt1 was remarkably decreased (Fig. 4a-c), which indicated that the Sirt1 knockdown was successful. Afterward, we found that miR-122 knockdown by transfecting miR-122 inhibitor into HepG2 or Huh-7 cells could potently decrease lipid deposition induced by FFA treatment; nevertheless, this inhibitory effect was abrogated by the co-transfection of shSirt1 (Fig. 4d). Moreover, miR-122 knockdown also restrained FFA-induced TG secretion; however, additional Sirt1 silencing could reverse this phenotype (Fig. 4e). These results evidenced that miR-122 inhibition could protect hepatocytes from excessive lipid deposition via the elevation ofSirt1 expression.

\section{miR-122 modulates lipogenic gene expression via targeting ofSirt 1}

To ascertain the underlying molecular mechanism of the regulation of intracellular lipid metabolism by miR-122, we measured the expression levels of major lipogenic genes, such as SREBP1, FASN, SCD1, ACC1 and ApoA5, in FFA-treated cells transfected with miR-122 inhibitor and/or shSirt1. qRT-PCR results showed that FFA treatment elevated the expression of these genes; however, their expression levels were suppressed by miR-122 inhibitor (Fig. 5). In addition, the co-transfection of miR-122 inhibitor and shSirt1 reinstated the expression of lipogenic genes at levels similar to those in cells treated solely with FFA (Fig. 5). These results clearly illustrated that miR-122 repressed Sirt1 expression, which then led to the upregulation of lipogenic genes and the consequential overproduction oflipids or steatosis in hepatocytes. Therefore, knockdown of miR-122 could effectively downregulate lipogenic genes via elevating Sirt1 expression in hepatocytes.

\section{Knockdown of miR-122 activates the LKB1/AMPK signalling pathway via enhancing Sirt1 expression} Previous studies have shown that the LKB1/AMPK pathway was implicated in the progression of NAFLD (Santamarina 


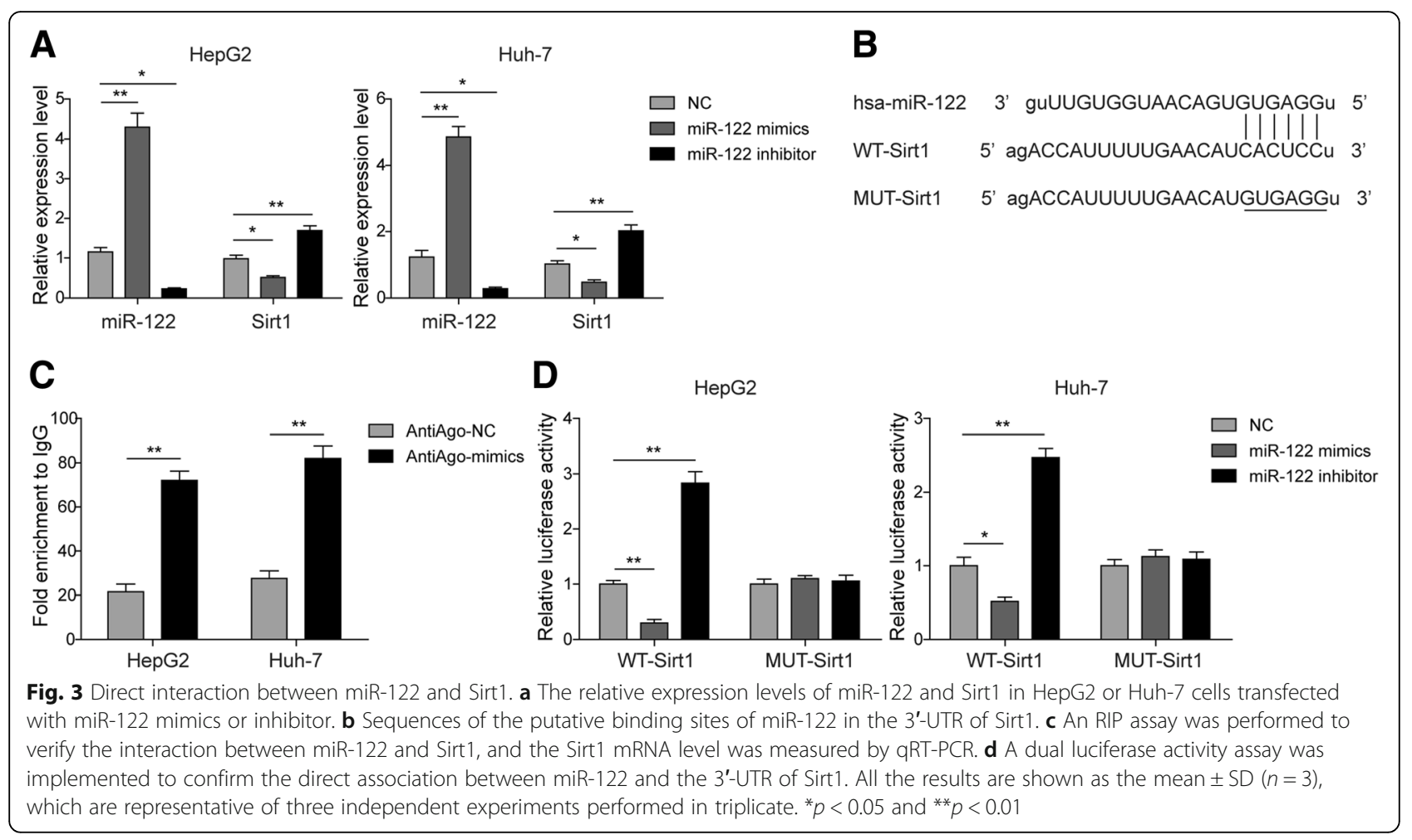

et al., 2015). Moreover, AMPK has been found to augment Sirt1 activity via elevating cellular $\mathrm{NAD}^{+}$levels (Canto et al., 2009), while Sirt1 overexpression also led to the activation of LKB1 and AMPK (Lan et al., 2008). To investigate whether the LKB1/AMPK pathway is required for the regulation of lipogenesis by miR-122 through Sirt1,we performed Western blotting to assess the influence of FFA treatment and miR-122 knockdown on the activation of the LKB1/ AMPK pathway. As expected, the result showed that FFA administration repressed the expression of Sirt1 and LKB1 as well as the phosphorylation of AMPK (p-AMPK) in HepG2 or Huh-7 cells. Nevertheless, the knockdown of miR-122 robustly upregulated LKB1 expression and significantly enhanced AMPK phosphorylation (Fig. 6a and b). In contrast, this expression pattern was again reversed by the co-transfection of shSirt1 (Fig. 6a and b). These results demonstrated that miR-122 suppressed Sirt1 expression, which further restrained the LKB1/AMPK signalling pathway.

\section{Discussion}

NAFLD is characterized by excessive storage of lipid droplets in hepatic tissues, whose normal physiological functioning is thus gradually compromised (Dai et al., 2017b). As a prevalent disease worldwide, NAFLD has resulted in tremendous costs for the healthy system, and severe steatohepatitis can even progress to liver cirrhosis or hepatocellular carcinoma (HCC). In this study, we first found that miR-122 was upregulated in NAFLD hepatic tissues/ cells, while Sirt1 was downregulated. After knocking down
miR-122 in HepG2 or Huh-7 cells by the transfection of miR-122 inhibitor, excessive lipid deposition was effectively rescued, which could be attributed to the upregulation of Sirt1 and the activation of the LKB1/AMPK signalling pathway. These findings thus provide new insights into the pathogenesis, diagnosis and therapyof NAFLD.

A recent study demonstrated that miRNAs, such as miR-17, miR-20a, miR-20b and miR-122, were closely associated with the initiation and progression of NAFLD (Ye et al., 2018). Due to the intrinsic function of miRNAs in modulating the expression of target genes, current studies mainly focus on the roles of miRNAs in the regulation of downstream genes, especially those involved in lipid metabolism. As reported, miR-24 has been found to promote hepatic lipid accumulation by suppressing the expression of insulin-induced gene 1 (Ng et al., 2014). Early studies have shown that miR-122 is a liver-specific miRNA and is closely associated with liver-related diseases (Alizadeh et al., 2015). In the present study, we found that miR-122 expression was remarkably elevated in both NAFLD mice and FFA-treated hepatocytes as determined by qRT-PCR. This result was consistent with a previous report that showed that there was an evident correlation between high miR-122 expression and NAFLD incidence in obese children (Brandt et al., 2018). Furthermore, several studies also confirmed a positive association between circulating miR-122 and histopathological features in NAFLD patients (Jampoka et al., 2018; Ye et al., 2018; Akuta et al., 


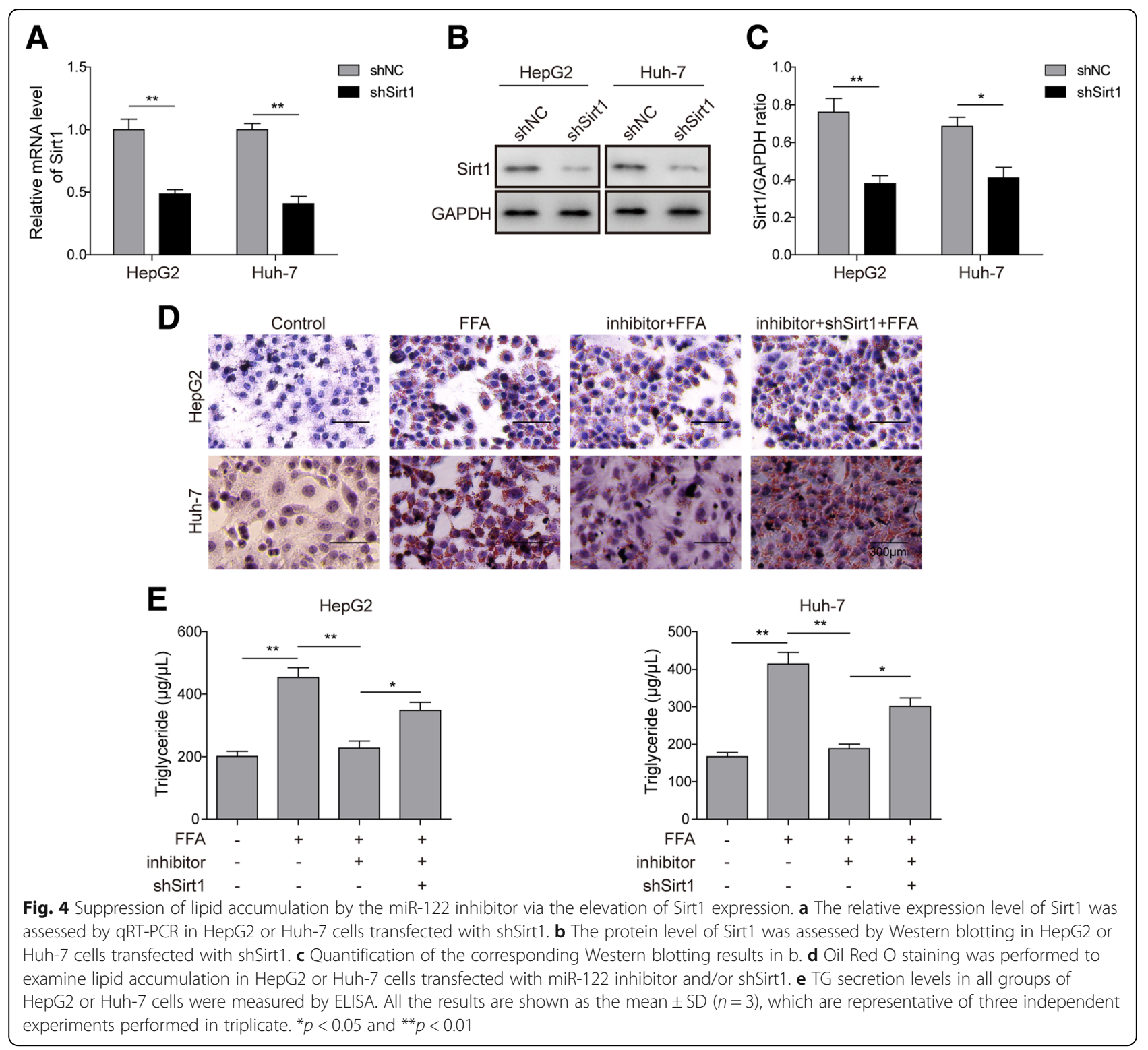

2016; Miyaaki et al., 2014). However, some previous studies showed that liver miR-122 levels were decreased while serum miR-122 levels were increased in human non-alcoholic steatohepatitis (Braza-Boils et al., 2016; Latorre et al., 2017; Sendi et al., 2018), which was not consistent with our results. We speculated that the different results might be due to the different experimental models and induction conditions of NAFLD, as well as the different severities of NAFLD patients. Overall, these previous studies and our results further substantiate the potency of miR-122 in the diagnosis and evaluation of NAFLD disease, although the underlying molecular mechanism still requires further investigation.

To identify the downstream target of miR-122 in our model, we first focused on Sirt1, which has been found to be downregulated in NAFLD patients (Castro et al.,
2013). A previous study also suggested that restoring Sirt1 expressioncould become a promising therapeutic strategy for NAFLD (Colak et al., 2014) due to the probable association between Sirt1 and lipid metabolic genes, such as SREBP1, FASN, and SCD1 (Wang et al., 2017; Sun et al., 2015). We thus investigated the possible involvement of miR-122 in regulating Sirt1 and its effect on the expression of lipogenic genes. Both RIP and dual luciferase reporter assays confirmed that Sirt1 was a direct target of miR-122 whose expression was repressed through the binding of miR-122 to its 3'-UTR and consequential mRNA degradation. The knockdown of Sirt1, on the other hand, abolished the suppressive effect of miR-122 inhibitor on lipogenesis. Moreover, multiple other miRNAs, such as miR-9 (Ao et al., 2016) and miR-34a (Kim et al., 2015), also target Sirt1 and facilitate 


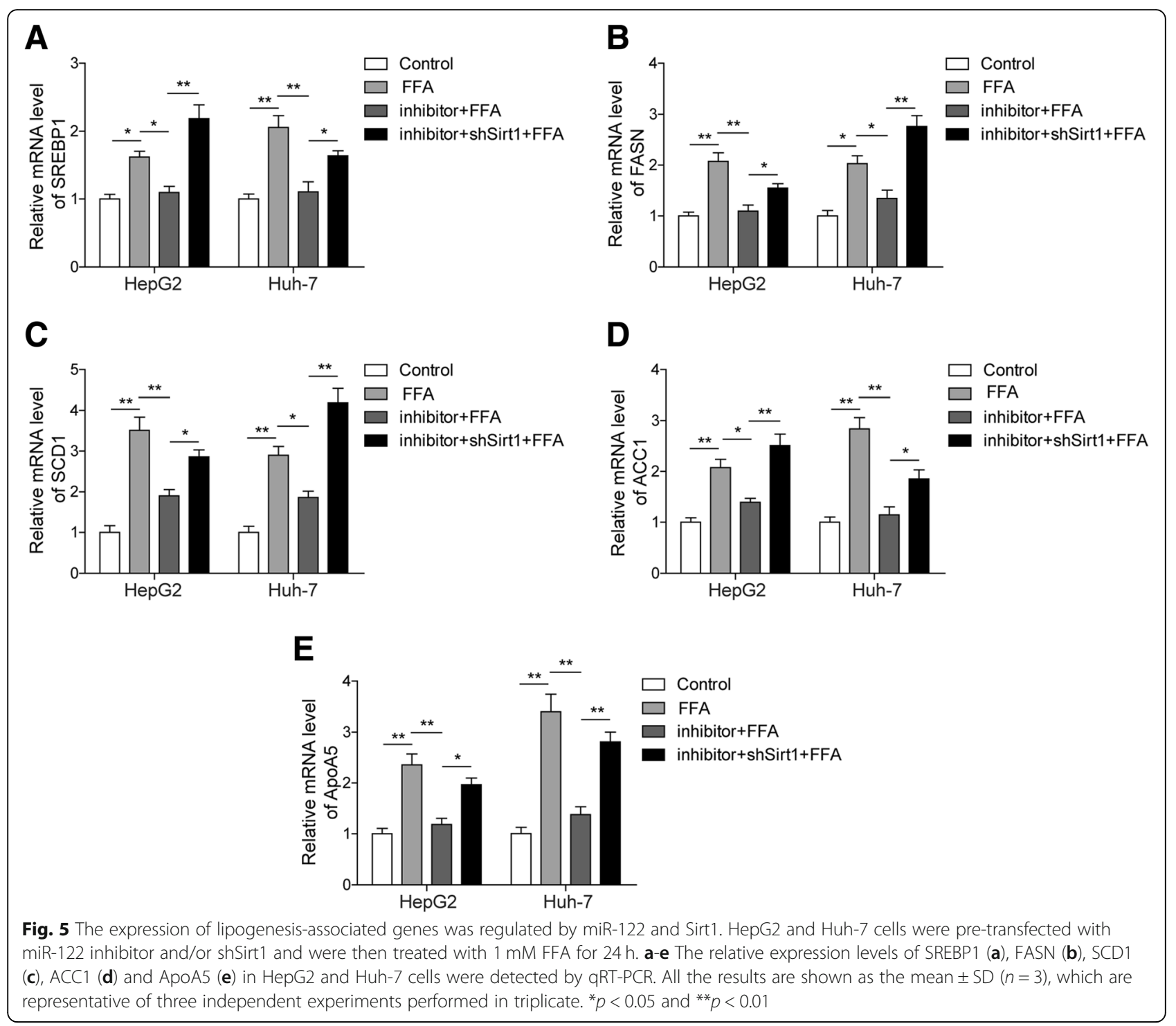

NAFLD progression via promoting lipogenesis. However, we first revealed that Sirt1 was a direct target of miR-122 and that miR-122 promoted NAFLD by directly inhibiting the expression of Sirt1.

NAFLD pathogenesis is closely related to lipid metabolic genes, including SREBP1, FASN, SCD1, ACC1 and ApoA5 (Lin et al., 2017; da Silva-Santi et al., 2016), whose expression are known to be regulated by distinct miRNAs. Until now, the role of miR-122 in modulating these genes and NAFLD progression has not been investigated. In this study, we further explored the functional effects of miR-122 in NAFLD. It was noted that miR-122 inhibition significantly decreased lipid accumulation and TG secretion in hepatocytes treated with FFA. In line with these results, one recent study reported that miR-122 played crucial roles in lipid droplet formation and TG secretion in hepatocytes under FFA-induced stress in culture medium (Wu et al., 2017). In addition, we examined the expression pattern of lipogenic genes in HepG2 and Huh-7 cells and found that they were prominently downregulated after transfection of the miR-122 inhibitor. These results showed that knockdown of miR-122 could alleviate NAFLD via suppressing the expression of lipid metabolic genes by directly upregulating Sirt1.

The LKB1/AMPK pathway is crucial for maintaining cellular homeostasis, especially for lipid metabolism (Lin et al., 2015). A previous study suggested that the LKB1/ AMPK axis was involved in liver pathology since its activation could prevent the occurrence of liver diseases (Santamarina et al., 2015). Moreover, LKB1 is believed to be downstream of Sirt1 and plays an indispensable role in ameliorating NAFLD conditions (Jia et al., 2016). As reported, LKB1 could suppress the expression of various 


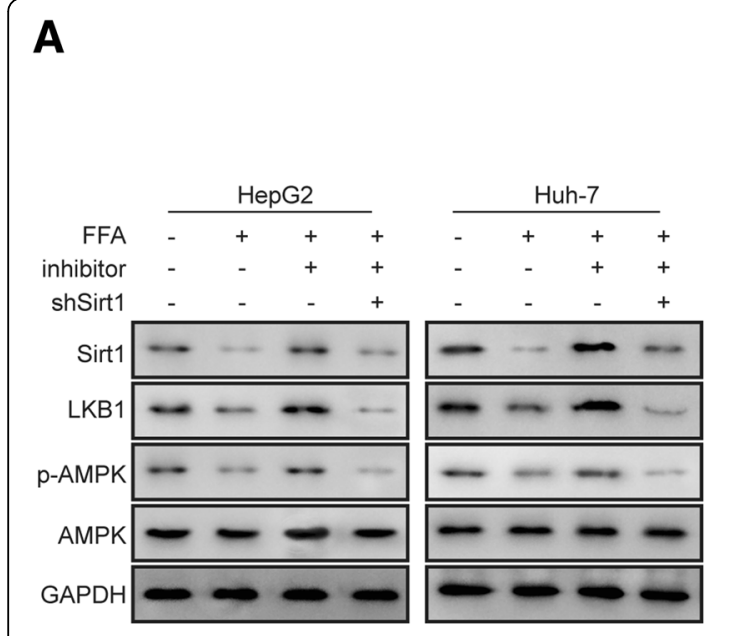

B
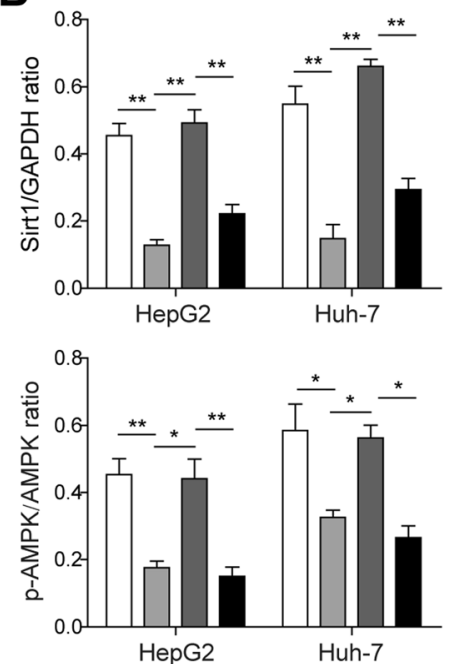

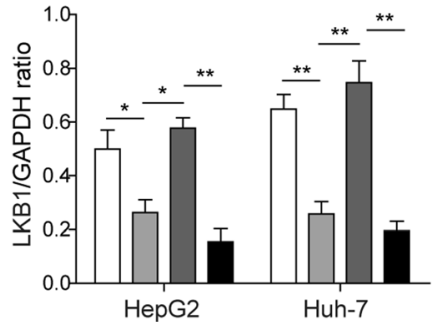

$\square$ Control

$\square$ FFA

$\square$ inhibitor+FFA

- inhibitor+shSirt1+FFA

Fig. 6 Downregulation of the LKB1/AMPK signalling pathway by the miR-122-Sirt1 axis. a The expression of Sirt1, LKB1, AMPK and the phosphorylation of AMPK ( $p$-AMPK) were measured by Western blotting in HepG2 or Huh-7 cells that were transfected with miR-122 inhibitor and/or shSirt1. b Quantification of the corresponding Western blotting results in a. All the results are shown as the mean \pm SD ( $n=3$ ), which are representative of three independent experiments performed in triplicate. ${ }^{*} p<0.05$ and ${ }^{* *} p<0.01$

lipogenesis-related genes, such as FASN and adiponectin (Gormand et al., 2014). In our experiments, we found that FFA treatment could suppress the activity of the LKB1/AMPK pathway, while miR-122 knockdown potently re-activated this pathway. These results indicated that the Sirt1/LKB1/AMPK pathway was responsible for the function of miR-122 in regulating lipid metabolism in hepatocytes. In addition, emerging evidence has demonstrated that tumour growth factor- $\beta$ (TGF- $\beta$ ) signalling, mammalian target of rapamycin complex 1 (mTOC1) signalling, Hedgehog signalling, c-Jun N-terminal kinase (JNK) signalling and MAPK signalling also participate in the regulation of lipid metabolism in the liver. However, only a few reports have explored the role of miR-122 in the modulation of these signalling pathways. It was reported that miR-122 and Sirt1 suppressed TGF- $\beta$ signalling to affect liver cancer metastasis (Yin et al., 2016) and ameliorate renal fibrosis (Huang et al., 2014), respectively. However, the association between the miR-122-Sirt1 axis and these signalling pathways in lipid metabolism has not yet been reported and requires further investigation.

\section{Conclusions}

In summary, this study found that miR-122 could repress the LKB1/AMPK signalling pathway via directly downregulating Sirt1, which then induced steatosis and lipogenesis in NAFLD. Therefore, knocking down miR-122 could re-activate the LKB1/AMPK axis, suppress lipid overproduction and alleviate NAFLD severity. These results revealed a novel function and detailed mechanism of miR-122 in regulating lipid metabolism in the liver, suggesting that miR-122 is a potential biomarker for NAFLD diagnosis and an appealing drug target for NAFLD therapy in the future.

\section{Abbreviations}

ACC1: Acetyl-coA carboxylase; AMPK: AMP-activated protein kinase; ApoA5: Apolipoprotein A5; FASN: Fatty acid synthase; FFA: Free fatty acid; HCC: Hepatocellular carcinoma; HE: Hematoxylin-eosin; HFD: High fat diet; IHC: Immunohistochemistry; JNK1: c-Jun N-terminal kinase 1; LKB1: Liver kinase B1; NAD: Nicotinamide adenine dinucleotide; NAFLD: Non-alcoholic fatty liver disease; $\mathrm{p}-\mathrm{AMPK}$ : AMPK phosphorylation; qRT-PCR: quantitative real-time PCR; RIP: RNA-immunoprecipitation; SCD: Standard chow diet; SCD1: Stearoyl-CoA desaturase 1; shRNA: short hairpin RNA; Sirt1: Sirtuin 1; SREBP1: Sterol regulatory element-binding protein 1; TG: Triglyceride; TGF$\beta$ : tumor growth factor- $\beta$; $\mathrm{mTOC} 1$ : mammalian target of rapamycin complex 1

\section{Acknowledgements}

We would like to give our sincere gratitude to the reviewers for their constructive comments.

\section{Funding}

Not applicable.

\section{Availability of data and materials}

All data generated or analyzed during this study are included in this published article [and its supplementary information files].

\section{Authors' contributions}

ZSP designed the study and reviewed the manuscript. LK designed the study, implemented literature research, performed experiments and edited the manuscript. DW performed experiments and prepared the manuscript. ZYW collected and analyzed data. All authors read and approved the final manuscript. 


\section{Ethics approval and consent to participate}

All animal experimental procedures followed the guideline of the Ethical Committee of the Second Xiangya Hospital, Central South University.

\section{Consent for publication}

Not applicable.

\section{Competing interests}

The authors declare that they have no competing interests.

\section{Publisher's Note}

Springer Nature remains neutral with regard to jurisdictional claims in published maps and institutional affiliations.

\section{Author details}

'Department of Cardiovascular Medicine, The Second Xiangya Hospital of Central South University, No.139, Middle Renmin Road, Changsha 410011, Hunan Province, People's Republic of China. ${ }^{2}$ Department of Urological Organ Transplantation, The Second Xiangya Hospital of Central South University, Changsha 410011, People's Republic of China.

\section{Received: 4 September 2018 Accepted: 17 April 2019}

Published online: 13 June 2019

\section{References}

Akuta N, Kawamura Y, Suzuki F, Saitoh S, Arase Y, Fujiyama S, Sezaki H, Hosaka T, Kobayashi M, Suzuki Y, Kobayashi M, Ikeda K, Kumada H. Analysis of association between circulating miR-122 and histopathological features of nonalcoholic fatty liver disease in patients free of hepatocellular carcinoma. BMC Gastroenterol. 2016;16:141.

Alizadeh E, Akbarzadeh A, Eslaminejad MB, Barzegar A, Hashemzadeh S, NejatiKoshki K, Zarghami N. Up regulation of liver-enriched transcription factors HNF4a and HNF6 and liver-specific microRNA (miR-122) by inhibition of let7b in mesenchymal stem cells. Chem Biol Drug Des. 2015;85:268-79.

Ao R, Wang Y, Tong J, Wang BF. Altered microRNA-9 expression level is directly correlated with pathogenesis of nonalcoholic fatty liver disease by targeting Onecut2 and SIRT1. Med Sci Monit. 2016;22:3804-19.

Brandt S, Roos J, Inzaghi E, Kotnik P, Kovac J, Battelino T, Cianfarani S, Nobili V, Colajacomo M, Kratzer W, Denzer C, Fischer-Posovszky P, Wabitsch M. Circulating levels of miR-122 and nonalcoholic fatty liver disease in prepubertal obese children. Pediatr Obes. 2018;13:175-82.

Braza-Boils A, Mari-Alexandre J, Molina P, Arnau MA, Barcelo-Molina M, Domingo D, Girbes J, Giner J, Martinez-Dolz L, Zorio E. Deregulated hepatic microRNAs underlie the association between non-alcoholic fatty liver disease and coronary artery disease. Liver Int. 2016;36:1221-9.

Canto C, Gerhart-Hines Z, Feige JN, Lagouge M, Noriega L, Milne JC, Elliott PJ, Puigserver P, Auwerx J. AMPK regulates energy expenditure by modulating NAD+ metabolism and SIRT1 activity. Nature. 2009:458:1056-60.

Castro RE, Ferreira DM, Afonso MB, Borralho PM, Machado MV, Cortez-Pinto H, Rodrigues CM. miR-34a/SIRT1/p53 is suppressed by ursodeoxycholic acid in the rat liver and activated by disease severity in human non-alcoholic fatty liver disease. J Hepatol. 2013;58:119-25.

Ceccarelli S, Panera N, Gnani D, Nobili V. Dual role of microRNAs in NAFLD. Int J Mol Sci. 2013;14:8437-55.

Chen X, Zhao M, Huang J, Li Y, Wang S, Harrington CA, Qian DZ, Sun XX, Dai MS. microRNA-130a suppresses breast cancer cell migration and invasion by targeting FOSL1 and upregulating ZO-1. J Cell Biochem. 2018;119:4945-56.

Chu MJ, Hickey AJ, Tagaloa S, Zhang L, Dare AJ, MacDonald JR, Yeong ML, Bartlett AS, Phillips AR. Ob/Ob mouse livers show decreased oxidative phosphorylation efficiencies and anaerobic capacities after cold ischemia. PLoS One. 2014;9:e100609.

Colak Y, Yesil A, Mutlu HH, Cakili OT, Ulasoglu C, Senates E, Takir M, Kostek O, Yilmaz Y, Enc FY, Tasan G, Tuncer I. A potential treatment of non-alcoholic fatty liver disease with SIRT1 activators. J Gastrointestin Liver Dis. 2014;23: $311-9$.

da Silva-Santi LG, Antunes MM, Caparroz-Assef SM, Carbonera F, Masi LN, Curi R, Visentainer JV, Bazotte RB. Liver fatty acid composition and inflammation in mice fed with high-carbohydrate diet or high-fat diet. Nutrients. 2016;8:682.

Dai H, Wang W, Chen R, Chen Z, Lu Y, Yuan H. Lipid accumulation product is a powerful tool to predict non-alcoholic fatty liver disease in Chinese adults. Nutr Metab (Lond). 2017b;14:49.
Dai W, Ye L, Liu A, Wen SW, Deng J, Wu X, Lai Z. Prevalence of nonalcoholic fatty liver disease in patients with type 2 diabetes mellitus: a meta-analysis. Medicine (Baltimore). 2017a;96:e8179.

Deng XQ, Chen LL, Li NX. The expression of SIRT1 in nonalcoholic fatty liver disease induced by high-fat diet in rats. Liver Int. 2007;27:708-15.

Gormand A, Berggreen C, Amar L, Henriksson E, Lund I, Albinsson S, Goransson $\mathrm{O}$. LKB1 signalling attenuates early events of adipogenesis and responds to adipogenic cues. J Mol Endocrinol. 2014;53:117-30.

Herranz D, Munoz-Martin M, Canamero M, Mulero F, Martinez-Pastor B, Fernandez-Capetillo O, Serrano M. Sirt1 improves healthy ageing and protects from metabolic syndrome-associated cancer. Nat Commun. 2010;1:3

Hu X, Zhang J, Jiang Y, Lei Y, Lu L, Zhou J, Huang H, Fang D, Tao G. Effect on metabolic enzymes and thyroid receptors induced by BDE- 47 by activation the pregnane $X$ receptor in $H e p G 2$, a human hepatoma cell line. Toxicol in Vitro. 2014;28:1377-85

Huang X-Z, Wen D, Zhang M, Xie Q, Ma L, Guan Y, Ren Y, Chen J, Hao C-M. Sirt1 activation ameliorates renal fibrosis by inhibiting the TGF- $\beta / S m a d 3$ pathway. J Cell Biochem. 2014;115:996-1005.

Imai S, Armstrong CM, Kaeberlein M, Guarente L. Transcriptional silencing and longevity protein Sir2 is an NAD-dependent histone deacetylase. Nature. 2000;403:795-800.

Jampoka K, Muangpaisarn P, Khongnomnan K, Treeprasertsuk S, Tangkijvanich P, Payungporn S. Serum miR-29a and miR-122 as potential biomarkers for nonalcoholic fatty liver disease (NAFLD). Microrna. 2018;7:215-22.

Jia L, Li W, Li J, Li Y, Song H, Luan Y, Qi H, Ma L, Lu X, Yang Y. Lycium barbarum. polysaccharide attenuates high-fat diet-induced hepatic steatosis by upregulating SIRT1 expression and deacetylase activity. Sci Rep. 2016;6:36209.

Kim HJ, Joe Y, Yu JK, Chen Y, Jeong SO, Mani N, Cho GJ, Pae HO, Ryter SW, Chung HT. Carbon monoxide protects against hepatic ischemia/reperfusion injury by modulating the miR-34a/SIRT1 pathway. Biochim Biophys Acta. 2015;1852:1550-9.

Lan F, Cacicedo JM, Ruderman N, Ido Y. SIRT1 modulation of the acetylation status, cytosolic localization, and activity of LKB1. Possible role in AMPactivated protein kinase activation. J Biol Chem. 2008;283:27628-35.

Latorre J, Moreno-Navarrete JM, Mercader JM, Sabater M, Rovira O, Girones J, Ricart W, Fernandez-Real JM, Ortega FJ. Decreased lipid metabolism but increased FA biosynthesis are coupled with changes in liver microRNAs in obese subjects with NAFLD. Int J Obes. 2017:41:620-30.

Li X. SIRT1 and energy metabolism. Acta Biochim Biophys Sin Shanghai. 2013;45: $51-60$.

Li X, Lian F, Liu C, Hu KQ, Wang XD. Isocaloric pair-fed high-carbohydrate diet induced more hepatic steatosis and inflammation than high-fat diet mediated by miR-34a/SIRT1 Axis in mice. Sci Rep. 2015;5:16774.

Lin MJ, Dai W, Scott MJ, Li R, Zhang YQ, Yang Y, Chen LZ, Huang XS. Metformin improves nonalcoholic fatty liver disease in obese mice via down-regulation of apolipoprotein A5 as part of the AMPK/LXRalpha signaling pathway. Oncotarget. 2017;8:108802-9.

Lin R, Elf S, Shan C, Kang HB, Ji Q, Zhou L, Hitosugi T, Zhang L, Zhang S, Seo JH, Xie J, Tucker M, Gu TL, Sudderth J, Jiang L, Mitsche M, DeBerardinis RJ, Wu S, Li Y, Mao H, Chen PR, Wang D, Chen GZ, Hurwitz SJ, Lonial S, Arellano ML, Khoury HJ, Khuri FR, Lee BH, Lei Q, Brat DJ, Ye K, Boggon TJ, He C, Kang S, Fan J, Chen J. 6-Phosphogluconate dehydrogenase links oxidative PPP, lipogenesis and tumour growth by inhibiting LKB1-AMPK signalling. Nat Cell Biol. 2015;17:1484-96.

Miyaaki H, Ichikawa T, Kamo Y, Taura N, Honda T, Shibata H, Milazzo M, Fornari F, Gramantieri L, Bolondi L, Nakao K. Significance of serum and hepatic microRNA-122 levels in patients with non-alcoholic fatty liver disease. Liver Int. 2014;34:e302-7.

Nassir F, Ibdah JA. Sirtuins and nonalcoholic fatty liver disease. World J Gastroenterol. 2016;22:10084-92.

$\mathrm{Ng} R$, Wu H, Xiao H, Chen X, Willenbring H, Steer CJ, Song G. Inhibition of microRNA-24 expression in liver prevents hepatic lipid accumulation and hyperlipidemia. Hepatology. 2014;60:554-64.

Panera N, Gnani D, Crudele A, Ceccarelli S, Nobili V, Alisi A. MicroRNAs as controlled systems and controllers in non-alcoholic fatty liver disease. World J Gastroenterol. 2014;20:15079-86.

Pirola CJ, Fernandez Gianotti T, Castano GO, Mallardi P, San Martino J, Mora Gonzalez Lopez Ledesma M, Flichman D, Mirshahi F, Sanyal AJ, Sookoian S. Circulating microRNA signature in non-alcoholic fatty liver disease: from serum non-coding RNAs to liver histology and disease pathogenesis. Gut. 2015;64:800-12. 
Purushotham A, Schug TT, Xu Q, Surapureddi S, Guo X, Li X. Hepatocyte-specific deletion of SIRT1 alters fatty acid metabolism and results in hepatic steatosis and inflammation. Cell Metab. 2009;9:327-38.

Rottiers V, Naar AM. MicroRNAs in metabolism and metabolic disorders. Nat Rev Mol Cell Biol. 2012;13:239-50.

Santamarina AB, Oliveira JL, Silva FP, Carnier J, Mennitti LV, Santana AA, de Souza GH, Ribeiro EB, Oller do Nascimento CM, Lira FS, Oyama LM. Green tea extract rich in Epigallocatechin-3-Gallate prevents fatty liver by AMPK activation via LKB1 in mice fed a high-fat diet. PLoS One. 2015;10:e0141227.

Sendi H, Mead I, Wan M, Mehrab-Mohseni M, Koch K, Atala A, Bonkovsky HL, Bishop CE. miR-122 inhibition in a human liver organoid model leads to liver inflammation, necrosis, steatofibrosis and dysregulated insulin signaling. PLoS One. 2018;13:e0200847.

Soares e Silva AK, de Oliveira Cipriano Torres D, dos Santos Gomes FO, dos Santos Silva B, Lima Ribeiro E, Costa Oliveira A, dos Santos LA, de Lima Mdo C, Pitta Ida R, Peixoto CA. LPSF/GQ-02 inhibits the development of hepatic steatosis and inflammation in a mouse model of non-alcoholic fatty liver disease (NAFLD). PLoS One. 2015;10:e0123787.

Spengler EK, Loomba R. Recommendations for diagnosis, referral for liver biopsy, and treatment of nonalcoholic fatty liver disease and nonalcoholic steatohepatitis. Mayo Clin Proc. 2015;90:1233-46.

Sun L, Wang Y, Song Y, Cheng XR, Xia S, Rahman MR, Shi Y, Le G. Resveratrol restores the circadian rhythmic disorder of lipid metabolism induced by high-fat diet in mice. Biochem Biophys Res Commun. 2015;458:86-91.

Takahashi Y, Sugimoto K, Inui H, Fukusato T. Current pharmacological therapies for nonalcoholic fatty liver disease/nonalcoholic steatohepatitis. World Gastroenterol. 2015;21:3777-85.

Wang LF, Wang XN, Huang CC, Hu L, Xiao YF, Guan XH, Qian YS, Deng KY, Xin HB. Inhibition of NAMPT aggravates high fat diet-induced hepatic steatosis in mice through regulating Sirt1/AMPKalpha/SREBP1 signaling pathway. Lipids Health Dis. 2017;16:82.

Wu GY, Rui C, Chen JQ, Sho E, Zhan SS, Yuan XW, Ding YT. MicroRNA-122 inhibits lipid droplet formation and hepatic triglyceride accumulation via Yin Yang 1. Cell Physiol Biochem. 2017;44:1651-64.

Yamada H, Ohashi K, Suzuki K, Munetsuna E, Ando Y, Yamazaki M, Ishikawa H, Ichino N, Teradaira R, Hashimoto S. Longitudinal study of circulating miR-122 in a rat model of non-alcoholic fatty liver disease. Clin Chim Acta. 2015;446: 267-71.

Ye D, Zhang T, Lou G, Xu W, Dong F, Chen G, Liu Y. Plasma miR-17, miR-20a, miR-20b and miR-122 as potential biomarkers for diagnosis of NAFLD in type 2 diabetes mellitus patients. Life Sci. 2018;208:201-7.

Yin S, Fan Y, Zhang H, Zhao Z, Hao Y, Li J, Sun C, Yang J, Yang Z, Yang X, Lu J, Xi $J$ J. Differential TGFbeta pathway targeting by miR-122 in humans and mice affects liver cancer metastasis. Nat Commun. 2016;7:11012.

Ready to submit your research? Choose BMC and benefit from:

- fast, convenient online submission

- thorough peer review by experienced researchers in your field

- rapid publication on acceptance

- support for research data, including large and complex data types

- gold Open Access which fosters wider collaboration and increased citations

- maximum visibility for your research: over $100 \mathrm{M}$ website views per year

At BMC, research is always in progress.

Learn more biomedcentral.com/submissions 